\title{
Isotropic and Uniaxial Strain Induced Band Modulation of PbTe
}

\author{
Xiaoguang Wang ${ }^{1, a}$, Yazhou Sun ${ }^{1, b}$, Liangming Peng ${ }^{1, c}$
}

\begin{abstract}
${ }^{1}$ CAS Key Laboratory for Mechanical Behavior and Design of Materials, Department of Modern Mechanics, School of Engineering Science, University of Science and Technology of China, Hefei 230026, PR China
\end{abstract}

aemail:wang2006@mail.ustc.edu.cn, bemail:yzsun@mail.ustc.edu.cn, cemail:penglm@ustc.edu.cn

Keywords: Strain; PbTe; First principles calculation; band structure

\begin{abstract}
First principles calculations were performed to explain the deformation feature and band gap variation behavior of PbTe under isotropic and uniaxial strain along [100], [110] and [111] directions. Calculations showed that the band gap variation regularity is quite different between the first and the last two cases due to the different lattice deformation modes. The introduction of shearing strain may significantly reduce the band gap of PbTe, and therefore the band structure of PbTe can be modulated by introducing proper strain.
\end{abstract}

\section{Introduction}

Thermoelectric (TE) materials have been attracted extensive attentions in the past two decades because of the realization of direct and environmental friendly conversion between heat energy and electronic power [1]. Generally, the performance of TE materials is determined by the dimensionless figure of merit $Z T=S^{2} \sigma T / \kappa$, where $S, \sigma, T$ and $\kappa$ are the Seebeck coefficient, electrical conductivity, absolute temperature and thermal conductivity, respectively. Accordingly, increasing the power factor, $P F=S^{2} \sigma$, is essential to enhancing $Z T$ [2-4]. Furthermore, the two related parameters, $S$ and $\sigma$, are both depended on the band structure [5].

Recently, strain engineering has been proved to be effective in tuning the band structure of many materials such as $\mathrm{Bi}_{2} \mathrm{Te}_{3}, \mathrm{Sb}_{2} \mathrm{Te}_{3}, \mathrm{Bi}_{2} \mathrm{O}_{2} \mathrm{Se}, \mathrm{La}_{2} \mathrm{NiO}_{4+\delta}$ and $\mathrm{SrTiO}_{3}$ to improve their TE properties [6-9]. Besides, this strain $(\varepsilon)$ effect has been also observed in lead telluride, which is one of the most potential and widely studied TE materials. Several studies have shown that the band gap of PbTe can be shifted by thermal or pressure induced strain [10-12] and the latter could even lead to a band inversion and topological phase transition $[13,14]$. However, such discussions on the band structure-strain relationship of PbTe are merely focused on the isotropic strain. PbTe is usually doped or designed into multiphase structures or 2D thin films to achieve better TE performance. Therefore, the actual strain or deformation conditions are far more complicated in most cases due to the heterogeneous doping and lattice mismatch [15-18]. In this contribution, discussions will be presented mainly dealing with the lattice deformation peculiarity and band structure evolutional behavior of PbTe under different types of strain.

\section{Computational details}

First principles calculations were performed using the projector augmented wave (PAW) [19] method and GGA-PBE exchange-correlation functional [20] as implemented in VASP [21]. Herein, discussions were focused on the tensile and compressive isotropic strain and uniaxial strain along [100], [110], [111] directions in PbTe. These strains were introduced basing on the conventional cell of PbTe with optimized lattice constant of $a_{0}=6.559 \AA$. The magnitude of strain was increased gradually in step of $1 \%$. Our calculations used $300 \mathrm{eV}$ plane wave cutoff energy and $11 \times 11 \times 11$ Monkhorst-Pack k-point sampling in the first Brillouin zone (BZ). The total energy converged within $1 \times 10^{-5} \mathrm{eV}$ and the interatomic forces were less than $0.01 \mathrm{eV} / \AA$ in all fully relaxed structures. The well known spin-orbit coupling (SOC) effect was included in all calculations except the structural relaxation since it almost exerts no influence on the structural optimization [17, 22]. 


\section{Results and discussions}

\section{Unstrained PbTe}

To begin with, some of the important features such as crystal and band structures of unstrained PbTe are summarized. PbTe normally exhibits a NaCl-type face-centered $(f c c)$ structure with lattice constant of $a=b=c=a_{0}$ and edge angle of $\alpha=\beta=\gamma=90^{\circ}$. In the simple cubic BZ of the conventional cell, both the valence band maximum (VBM) and conduction band minimum (CBM) exist at the $\boldsymbol{R}$ point and generate a direct band gap $\left(E_{g}\right)$. The calculated $E_{g}$ is $0.077 \mathrm{eV}$, which consists well with the previously calculated results [22, 23].

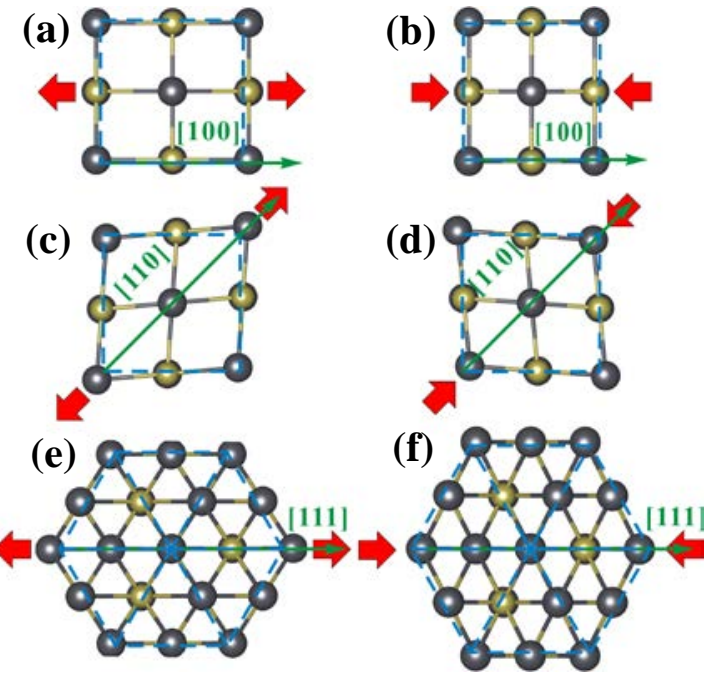

Fig. 1. Relaxed structures of $\mathrm{PbTe}$ with $10 \%$ tensile and compressive strain along [100], [110] and [111] directions. Unstrained lattice is given by dashed lines.

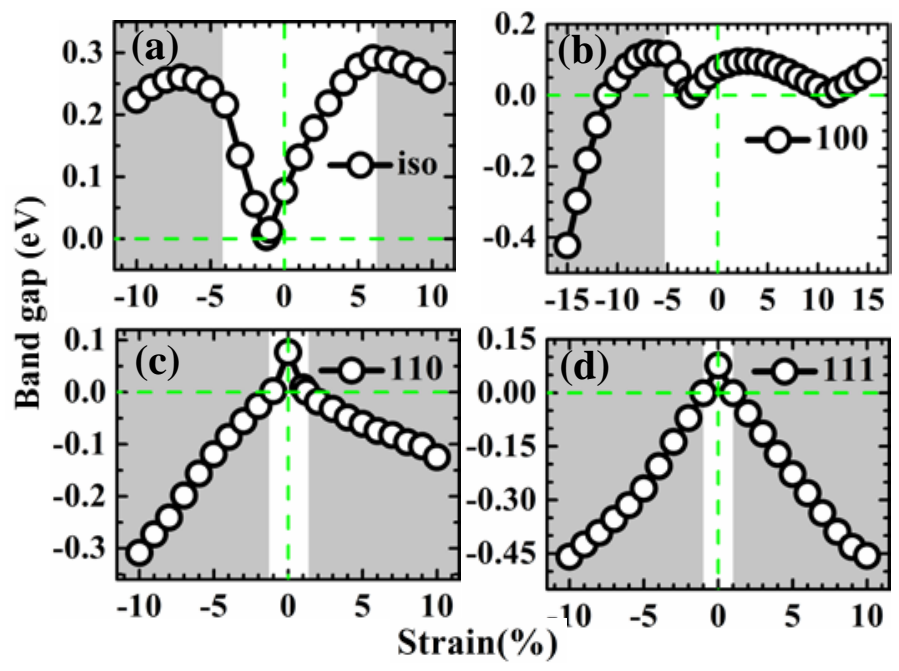

Fig. 2. The band gap variation of PbTe with the strength of (a) isotropic strain and uniaxial strain along (b) [100], (c) [110] and (d) [111] directions. Band gaps in grey areas are indirect ones.

\section{Structural evolution}

$\mathrm{PbTe}$ with isotropic strain maintains the $f c c$ symmetry during the deformation. However, as illustrated in Fig. 1, situations under uniaxial strains are more complex. It is reasonable that tensile strain along [100] direction induces a reduction of $b$ and $c$ because of the Poisson effect, whereas it behaves just oppositely in compressive condition (cf. Figs. 1 (a) and (b)). In these two cases, the three orthogonal directions, $\boldsymbol{a}, \boldsymbol{b}$ and $\boldsymbol{c}$, remain mutually perpendicular during the deformation, resulting in a cubic-tetragonal lattice transition trend.

Fig. 1 (c) depicts the relaxed structure with tensile strain along [110] direction. In this case, both $a$ and $b$ are elongated whereas $c$ is decreased. Furthermore, the original right angle $\gamma$ is reduced significantly. Again, the compressive strain produces an opposite effect (Fig. 1 (d)). In comparison, the [111] directional strain induces more symmetric lattice deformation. Concretely, the tensile strain results in a lattice constant of $a=b=c>a_{0}$ and edge angle of $\alpha=\beta=\gamma<90^{\circ}$ while compressive strain leads to $a=b=c<a_{0}$ and $\alpha=\beta=\gamma>90^{\circ}$, as evident in Figs. 1 (e) and (f). Nevertheless, such angle change (shearing strain) does not exist in the isotropically and [100] directionally strained conditions although the lattice constants change similarly.

\section{Band structure}

Fig. 2 shows the strain-dependence of band gap in PbTe. $E_{g}$ increases firstly and then decreases with the increase of isotropic tensile strain and the maximum value of $0.292 \mathrm{eV}$ occurs at $\varepsilon=6 \%$ (Fig. 2 (a)). It is worth noting that a direct-to-indirect band gap transition is observed for $\varepsilon>6 \%$. This is mainly attributed to the down-shift of the light $\mathbf{L}$ band and the slight up-shift of the heavy $\boldsymbol{\Sigma}$ band, inducing the convergence of valence band that is beneficial to the thermoelectric performance of $\mathrm{PbTe}[24,25]$. In compressive condition, $E_{g}$ is decreased at first and then closed at $\varepsilon \approx-1.20 \%$. 
Intriguingly, the band gap is reopened and increased with further increasing the compressive strain. The observed phenomena are consistent well with the band gap variation regularity of PbTe under pressure [11, 12, 26]. It should be noted that the band gap becomes indirect for $\varepsilon<-4 \%$ and $E_{g}$ begins to decrease again for $\varepsilon<-7 \%$.

From Fig. 2 (b), it can be seen that the band gap of PbTe with [100] uniaxial strain changes in a similar trend to that under isotropic condition in the strain region of $-10 \% \leq \varepsilon \leq 10 \%$. However, tensile strain did not lead to indirect band gap transition and $E_{g}$ varied more gently in the former. The similarity might be originated from the deformation similitude that only linear strain were introduced in both cases. It is worth noting that the band gap can be closed when [100] tensile strain reaches $11 \%$ and reopened by further increase the strain magnitude. However, $E_{g}$ continues to reduce after reaching its maximum value of $0.120 \mathrm{eV}$ at $\varepsilon=-7 \%$ in the compressive condition and reclosed at $\varepsilon=-11 \%$. Moreover, the CBM and VBM overlaps for $\varepsilon<-11 \%$, resulting in negative band gaps and a metal transition. Accordingly, the change of band gap with strain exhibits strong tension-compression asymmetry under the isotropically and [100] directionally strained conditions.

As shown in Figs. 2 (c) and (d), quite different band gap changing regularities were observed under [110] and [111] directionally strained conditions due to the shearing strain. In both cases, $E_{g}$ decreases monotonously with the increase of tensile and compressive strain. The band gap is closed at $\varepsilon=1.23 \%$ and $-1 \%$ in the [110] case and the corresponding values are $\pm 1 \%$ in [111] condition. After the closure, the valence and conduction bands are overlapped and indirect negative band gaps are generated. Please note that the [111] directional strain induced variation of $E_{g}$ are almost the same in tensile and compressive cases. The aforementioned symmetric lattice deformation should be responsible for this tension-compression symmetry of the band gap variation.

\section{Conclusions}

In summary, the deformation peculiarity and band gap variation behavior of $\mathrm{PbTe}$ under isotropic and typical uniaxial strain were investigated by first principles calculations. The preliminary results reveal that the lattice edge angle of PbTe can be changed by [110] and [111] directional linear strain, and thus shearing strain is introduced. Due to the lattice distortion, the band gap decreases monotonously with increasing tensile and compressive strain, which is completely different from that in the isotropically and [100] uniaxially strained conditions. However, band gap closure is observed in all cases. Accordingly, the lattice deformation type is dominant in modulating the band structure of PbTe. Therefore, one may tune the band structure of such materials intentionally by introducing proper strain to achieve higher $Z T$ for practical applications.

\section{Acknowledgements}

Authors thank the Supercomputing Center (SCC) of University of Science and Technology of China (USTC) for the computing facility supports.

\section{References}

[1] L.E. Bell. Cooling, heating, generating power, and recovering waste heat with thermoelectric systems [J]. Science, 2008321 (5895) 1457-1461.

[2] M. Zebarjadi, K. Esfarjani, Z.X. Bian, et al. Low-Temperature Thermoelectric Power Factor Enhancement by Controlling Nanoparticle Size Distribution [J]. Nano Letters, 201111 (1) 225-230.

[3] J.H. Bahk, P. Santhanam, Z.X. Bian, et al. Resonant carrier scattering by core-shell nanoparticles for thermoelectric power factor enhancement [J]. Applied Physics Letters, 2012100 (1) 012102.

[4] B. Paul, P.K. Rawat, P. Banerji. Dramatic enhancement of thermoelectric power factor in PbTe:Cr co-doped with iodine [J]. Applied Physics Letters, 201198 (26) 262101.

[5] J.R. Sootsman, D.Y. Chung, M.G. Kanatzidis. New and Old Concepts in Thermoelectric 
Materials [J]. Angewandte Chemie-International Edition, 200948 (46) 8616-8639.

[6] D. Zou, Y. Liu, S. Xie, et al. Effect of strain on thermoelectric properties of SrTiO3: First-principles calculations [J]. Chemical Physics Letters, 2013586 159-163.

[7] V. Pardo, A.S. Botana, D. Baldomir. Strain effects to optimize thermoelectric properties of hole-doped $\mathrm{La}_{2} \mathrm{NiO}_{4+\delta}$ via ab initio calculations [J]. Physical Review B, 201387 (12) 125148.

[8] D. Guo, C. Hu, Y. Xi, et al. Strain Effects To Optimize Thermoelectric Properties of Doped Bi2O2Se via Tran-Blaha Modified Becke-Johnson Density Functional Theory [J]. The Journal of Physical Chemistry C, 2013117 (41) 21597-21602.

[9] N.F. Hinsche, B.Y. Yavorsky, I. Mertig, et al. Influence of strain on anisotropic thermoelectric transport in $\mathrm{Bi}_{2} \mathrm{Te}_{3}$ and $\mathrm{Sb}_{2} \mathrm{Te}_{3}$ [J]. Physical Review B, 201184 (16) 165214.

[10] J.W. Tomm, L. Werner, D. Genzow, et al. Experimental evidence of strain-induced gap shifts in PbTe epitaxial layers by photoluminescence [J]. Physica Status Solidi (a), 1988106 (2) 509-514.

[11] Y.L. Yang. Elastic moduli and band gap of PbTe under pressure: ab initio study [J]. Materials Science and Technology, 201228 (11) 1308-1313.

[12] L.Q. Xu, Y.P. Zheng, J.C. Zheng. Thermoelectric transport properties of PbTe under pressure [J]. Physical Review B, 201082 (19) 195102.

[13] P. Barone, T. Rauch, D. Di Sante, et al. Pressure-induced topological phase transitions in rocksalt chalcogenides [J]. Physical Review B, 201388 (4) 045207.

[14] W. Feng, W. Zhu, H.H. Weitering, et al. Strain tuning of topological band order in cubic semiconductors [J]. Physical Review B, 201285 (19) 195114.

[15] K. Biswas, J.Q. He, Q.C. Zhang, et al. Strained endotaxial nanostructures with high thermoelectric figure of merit [J]. Nature Chemistry, 20113 (2) 160-166.

[16] K. Biswas, J.Q. He, I.D. Blum, et al. High-performance bulk thermoelectrics with all-scale hierarchical architectures [J]. Nature, 2012489 (7416) 414-418.

[17] X.G. Wang, J. Liu, L.M. Peng. First-principles investigation of dual substitutional impurity-induced electronic structural modulation of PbTe on cationic and anionic sites [J]. Acta Materialia, 201361 (17) 6428-6442.

[18] R. Leitsmann, F. Bechstedt. Electronic-structure calculations for polar lattice structure mismatched interfaces: PbTe/CdTe(100) [J]. Physical Review B, 200776 (12) 125315.

[19] P.E. Blochl. PROJECTOR AUGMENTED-WAVE METHOD [J]. Physical Review B, 199450 (24) 17953-17979.

[20] J.P. Perdew, K. Burke, M. Ernzerhof. Generalized Gradient Approximation Made Simple [J]. Physical Review Letters, 199677 (18) 3865-3868.

[21] G. Kresse, J. Furthmuller. Efficient iterative schemes for ab initio total-energy calculations using a plane-wave basis set [J]. Physical Review B, 199654 (16) 11169-11186.

[22] Y. Zhang, X.Z. Ke, C.F. Chen, et al. Thermodynamic properties of PbTe, PbSe, and PbS: First-principles study [J]. Physical Review B, 200980 (2) 024304.

[23] K. Hoang, S.D. Mahanti, M.G. Kanatzidis. Impurity clustering and impurity-induced bands in PbTe-, SnTe-, and GeTe-based bulk thermoelectrics [J]. Physical Review B, 201081 (11) 115106.

[24] Y. Pei, X. Shi, A. LaLonde, et al. Convergence of electronic bands for high performance bulk thermoelectrics [J]. Nature, 2011473 (7345) 66-69.

[25] L.D. Zhao, H.J. Wu, S.Q. Hao, et al. All-scale hierarchical thermoelectrics: MgTe in PbTe facilitates valence band convergence and suppresses bipolar thermal transport for high performance [J]. Energy \& Environmental Science, 20136 (11) 3346-3355.

[26] A. Svane, N.E. Christensen, M. Cardona, et al. Quasiparticle self-consistent GW calculations for PbS, PbSe, and PbTe: Band structure and pressure coefficients [J]. Physical Review B, 201081 (24) 245120. 